\title{
Gestão da qualidade hospitalar na assistência dos serviços de enfermagem
}

\author{
Poliana Nunes Gesteira ${ }^{1}$ \\ Henry Maia Peixoto ${ }^{2}$ \\ Hélia Carla de Souza ${ }^{3}$ \\ Luciana Farias de Miranda ${ }^{4}$ \\ Lígia Richter de Alencar Normando ${ }^{5}$
}

\section{Resumo}

Este trabalho tem como objetivo dissertar sobre alguns dos fatores da qualidade, no que se refere à estrutura, processo e resultado dos serviços de enfermagem no hospital que influenciam na qualidade da assistência prestada ao cidadão. Trata-se de um estudo bibliográfico de caráter descritivo, e foram utilizados como dados artigos científicos publicados no período de 1990 a 2009. A busca pela qualidade sempre esteve presente. O que levou as empresas industriais a optarem por serviços de qualidade foi a competição que estava acontecendo entre elas. Foi criado o Programa de Gestão pela Qualidade Total para auxiliar as empresas a gerenciarem com qualidade. Os hospitais são avaliados pela Joint Commission on Accreditation of Hospitals e pela Organização Nacional de Acreditação. Ambas avaliam de acordo com o proposto por Donabedian: estrutura, processo e resultado. A acreditação inclui avaliação da qualidade da assistência de enfermagem.

Palavras-chave: Gestão. Qualidade. Enfermeiro. Avaliação da qualidade. Satisfação dos usuários.

1 Graduada em Enfermagem.

2 Especialista em Saúde do Adolescente; Especialista em Saúde da Família; Professor do Curso de Enfermagem do UniCEUB e Faculdade LS.

3 Especialista em Docência do Ensino Superior; Professora do Curso de Enfermagem do UniCEUB.

4 Especialista em Gestão do Programa Saúde da Família. Professora da Faculdade de Enfermagem do Centro Universitário UNIEURO.

5 Mestre em Ciência da Reabilitação, Professora do Curso de Enfermagem do UniCEUB. 


\section{Introdução}

Dr. Crooks conseguira eliminar o mau cheiro dos hospitais com uma substância química chamada fenol. Robert Koch, com o microscópio criado por Galileu, evidenciou a existência de micróbios e, em 1880, foram descobertos os germes (MELLO; CAMARGO, 1998).

A clínica também evoluía. Surgiu a insulina para os diabéticos, o extrato hepático para anemia perniciosa e imunizações específicas. Enfim, essas evoluções aconteceram e a busca pela qualidade esteve presente, já que os problemas existiam e as pessoas pensavam em como melhorá-los ou resolvê-los. Sendo assim, procuravam soluções observando, pesquisando, trocando ideias, experimentando, inovando, analisando resultados, arquivando as documentações e melhorando resultados obtidos (MELLO; CAMARGO, 1998).

Durante a década de 1980, havia muitas empresas industriais, principalmente nos Estados Unidos e Japão. Diante disso, começou a existir uma grande competição, e com isso a necessidade de adotar programas sistemáticos de qualidade, aplicando, assim, a gestão da qualidade não somente na produção, como também em todos os departamentos. Para algumas empresas, mudar a maneira de administrar foi uma questão de sobrevivência (FALCONI, 2004).

Desde o século XX, a única forma de "gestão da Qualidade" foi a padronizada por Taylor: a inspeção. Inspecionar, para ele, era treinar pessoas para vistoriar os serviços dos trabalhadores, avaliando os resultados como forma de aumentar a produtividade e a satisfação do cliente. A inspeção adicionava custos, porém, melhorava a qualidade dos serviços (BERWICK; GODFREY; ROESNER, 1994).

Walter Shewhart estudou mais sobre a inspeção supracitada, e declarou que é muito mais eficiente reparar os problemas nos processos de produção, do que reparar os problemas somente nos produtos (resultados), desenvolvendo assim, o método de controle da qualidade (BERWICK; GODFREY; ROESNER, 1994).

Mais tarde, os japoneses ampliaram esse conceito, incluindo a participação de toda força de trabalho na administração da qualidade, independentemente 
do nível hierárquico. Dessa forma, o controle de Qualidade Total foi o programa proposto com mais êxito, e as empresas que zelam por bons serviços gerenciam seguindo esse modelo (BARROS, 2005).

A área de saúde, ainda que de forma lenta, despertou para a Qualidade nos anos 80 , devido aos recursos financeiros cada vez menores e aos custos cada vez maiores, além de uma variada gama de pressões vindas do governo, da indústria e dos usuários. Essa nova situação tem feito algumas instituições da área de saúde reavaliarem sua forma de administração e adotarem o Gerenciamento da Qualidade (ANTUNES; TREVIZAN, 2000).

Quando se fala em Qualidade em saúde, pode-se citar Florence Nightingale, enfermeira inglesa que, quando pensava em agrupar os pacientes mais graves e com maior grau de dependência em um setor onde a enfermagem pudesse manter uma vigilância e atendimento constantes, ar e água limpos, controle de ruídos, e as formas pelas quais estes afetavam a recuperação do paciente, estava vivenciando o modelo de melhoria de Qualidade contínua em saúde (NOGUEIRA, 1999).

A enfermagem, a exemplo dos hospitais, começa a estudar práticas específicas para adotar o Gerenciamento da Qualidade tendo como objetivo alcançar não só um padrão aceitável de assistência, mas também a atender as expectativas dos trabalhadores e dos pacientes (ANTUNES; TREVIZAN, 2000).

Para avaliar a Qualidade, foram produzidas inúmeras pesquisas sobre o assunto, desenvolvendo a tríade de avaliação baseada em estrutura, processo e resultado (BARROS, apud DONABEDIAN, 1994).

Entende-se por estrutura: os recursos materiais, humanos, financeiros e os aspectos organizacionais. Já o processo está relacionado ao que se faz pelo e para o usuário, incluindo a Qualidade e a habilidade dos serviços prestados. E a avaliação do resultado demonstra o que é alcançado pelo e para o usuário, seja ele um indivíduo ou um grupo (DONABEDIAN, 1991). 
O gerenciamento da Qualidade pode ser útil à enfermagem, ajudando-a a superar as dificuldades rotineiras, a incorporar novos métodos e técnicas, a realizar as mudanças necessárias ao seu desenvolvimento e a alcançar a Qualidade da assistência (ANTUNES; TREVIZAN, 2000).

Pensar na gestão da qualidade da assistência de enfermagem é a vontade de querer cuidar visando a satisfação do cliente como questão primordial do processo de qualidade (KURCGANT et al, 2005).

Motivados por esse desafio, os autores optaram por ter como objetivo deste trabalho, descrever alguns dos fatores de qualidade, no que se refere à avaliação dos serviços de enfermagem, que influenciam na qualidade da assistência prestada ao cidadão, visando à assistência como preocupação constante na atualidade pelas instituições que almejam bons serviços para a sociedade.

\section{Metodologia}

Para a elaboração desta pesquisa bibliográfica, foram consultadas diversas fontes no período de 1990 a 2009, tais como: livros, trabalhos acadêmicos, artigos publicados em periódicos nacionais e internacionais. As palavras chaves utilizadas para procurar os artigos foram: Gestão, Qualidade, Enfermeiro, Avaliação da Qualidade, Satisfação dos Usuários. Foram analisados vinte e cinco livros, quatro teses de doutorado, uma dissertação de mestrado e uma extensa revisão de literatura de fontes online nacionais e internacionais. $\mathrm{O}$ estudo incluiu toda a literatura sobre o tema identificada no período estabelecido.

\section{Resultados}

A evolução da qualidade passou por três grandes processos: inspeção, controle estatístico e qualidade total. $\mathrm{Na}$ era da inspeção, o produto era examinado pelo produtor e pelo cliente, o que ocorreu pouco antes da revolução industrial. Os principais responsáveis pela inspeção eram os próprios artesãos, denominados 
inspetores, por Frederick Taylor. Nessa época, a preocupação encontrava-se no produto - resultado de um esforço organizado (OLIVEIRA et al., 2004).

$\mathrm{Na}$ era seguinte (controle estatístico), o controle da inspeção foi aprimorado por meio de utilização de técnicas específicas. Essa nova maneira de administrar obedecia a cálculos estatísticos, em que se selecionavam os produtos aleatoriamente, podendo verificar a qualidade de todo o lote dos produtos, isto é, procuravam aperfeiçoar a "construção" do trabalho (processo), para a obtenção de um bom resultado. Nessa época, tinham enfoque no processo, ou seja, nas atividades realizadas pela equipe (OLIVEIRA et al., 2004).

E na era da Qualidade Total, na qual se enquadram os dias atuais, a ênfase passa a ser dada às pessoas (clientes internos e externos), atendendo às questões de qualidade na área de serviços, intensificando-se a busca de mudanças, tanto comportamental, quanto cultural dos indivíduos envolvidos no processo, objetivando, desse modo, a satisfação do cliente (KURCGANT et al., 2005).

Qualquer que seja o tipo de organização - hospital, escola, fábrica - a competição é algo que sempre estará presente. As transformações ocorridas no mundo como a globalização da economia, difusão de novas tecnologias e a socialização dos meios de comunicação, são alguns eventos que contribuíram para a mudança do comportamento dos usuários dos serviços, aumentando dessa forma a competitividade entre as organizações (KURCGANT et al., 2005).

E como aconteceu a evolução da qualidade hospitalar? O Colégio Americano de Cirurgiões (CAC), em meados de 1924, estabeleceu o Programa de Padronização Hospitalar - PPH. Eles acreditavam que, para garantir a qualidade para os pacientes, eram necessários três padrões: corpo médico bem organizado, preenchimento do prontuário completo (incluindo anamnese, exame físico e alta hospitalar) e existência de recursos diagnóstico e terapêutico (o hospital deveria ter no mínimo um laboratório clínico e um departamento radiológico) (FELDMAN; GATTO; CUNHA, 2005).

Porém, após avaliarem os serviços, puderam perceber que a realidade era bem diferente, e se deram conta de que somente esses padrões não seriam sufi- 
cientes para proporcionarem qualidade ao paciente, pois não levaram em consideração outros aspectos importantíssimos: dimensionamento de pessoal de enfermagem, necessidade da assistência durante as 24 horas do dia, estrutura física do hospital adequada, avaliação dos resultados com o paciente, entre outros. (VARGAS et al., 2007).

Em 1951, foi criada a Comissão conjunta dos hospitais, que um ano depois foi delegada oficialmente como o Programa de Acreditação Hospitalar: a Joint Commission on Accreditation of Hospitals (FELDMAN; GATTO; CUNHA, 2005).

A Acreditação Hospitalar é um prêmio a que as empresas que estão inseridas concorrem. Este é com frequência um processo voluntário do qual as organizações decidem participar. Os padrões de Acreditação costumam considerar como ótimos e factíveis e têm sido desenhados para obter uma melhoria contínua e crescente das organizações acreditadas (VARGAS et al., 2007).

Acreditar é sinônimo de aprovar oficialmente. Para tanto, utilizam-se critérios de avaliação da estrutura, processos e resultados. O oficial Acreditador é a JCAHO - Comissão Conjunta de Acreditação de Organizações de Assistência à Saúde (Joint Commission on Acreditation of Health Care Organization). E, em 1946, foi legitimado o Certificado ISO (International Standart Organization). Ambos são mecanismos que têm como objetivo desencadear um determinado padrão mundial de qualidade (NOGUEIRA, 1999).

No Brasil existe a Organização Nacional de Acreditação (ONA), que é um programa de Acreditação Hospitalar, criado pelo Ministério da Saúde em 1997, com o objetivo de ter um padrão de qualidade hospitalar compatível com uma referência externa (VARGAS et al., 2007)

Essa organização classifica os hospitais, de acordo com a avaliação, em três níveis. As exigências do Nível um estão relacionadas com o atendimento aos requisitos básicos da qualidade na assistência. O Nível dois contempla a adoção do planejamento na organização da assistência hospitalar. O Nível três refere-se às políticas de melhorias contínuas (LIMA; ERDMANN, 2006). 
Para se alcançar o Nível um nos serviços de enfermagem, é necessária a presença do responsável técnico habilitado, supervisão contínua e sistematizada; dimensionamento de pessoal adequado para as 24 horas do dia; evolução e anotação de enfermagem completas no prontuário, além de auditoria de prontuário e controle dos pacientes internados (VARGAS et al., 2007).

No Nível dois destacam-se: disposição de manuais de procedimentos dos serviços de enfermagem padronizados para todas as unidades; programas de educação continuada; comissões voltadas para a melhoria do atendimento dos serviços de enfermagem; presença dos indicadores de qualidade de enfermagem e prática da Sistematização da Assistência dos Serviços de Enfermagem (VARGAS et al., 2007)

O Nível três refere-se a estar integrado no programa da qualidade e produtividade; realizar "plano de metas com resultados numéricos" e classificá-los como metas atingidas, não atingidas e em andamento; aperfeiçoamento da qualidade assistencial; e, indicador de nível de satisfação dos clientes internos e externos (VARGAS et al., 2007; NOGUEIRA, 1999).

É importante ressaltar que paciente é sinônimo de cliente externo. Os clientes externos são aqueles que não pertencem à instituição e que utilizam os serviços ou compram os mesmos; existem também os clientes internos que são as pessoas que trabalham na instituição e que necessitam dos serviços que ela executa, por exemplo: a enfermagem necessita da agilidade da farmácia, que precisa dos materiais do almoxarifado, entre outros). O cliente, seja ele interno ou externo, deve ter suas necessidades atendidas (MEZOMO, 1995).

O hospital tem como desafio satisfazer às necessidades e desejos fundamentais dos clientes. Com isso, a unidade hospitalar deve prestar um serviço de qualidade em todas as suas dimensões (MEZOMO, 1995).

Todavia, o hospital não é como uma indústria de automóveis, pois envolve o processo do cuidado com vidas que se encontram sob riscos. Logo, gerenciar esse processo é mais delicado do que se imagina (COCK; HIPKIN, 1997). 
O trabalho de enfermagem, assim como o trabalho de saúde, integra prestação de serviços à saúde, não produz bens a serem adotados e comercializados, e sim serviços que são consumidos no ato de sua produção, ou seja, no momento da assistência. Logo, é um trabalho diferenciado por lidar com o ser humano (KURCGANT et al, 2005).

Pensando nisso, o serviço de enfermagem organizou-se em três direções: na organização do cuidado com o doente, cuidando do paciente por meio de técnicas de enfermagem; na organização do ambiente, por mecanismos de limpeza, higiene; e na organização dos agentes de enfermagem, com a educação continuada. Dessa forma, a enfermagem assume, no ambiente hospitalar, um papel tanto assistencial quanto gerencial (KURCGANT et al., 2005).

Um dos métodos utilizados é o Controle da Qualidade Total que tem como objetivo produzir e fornecer produtos de que o cliente esteja necessitando; conseguir ter lucro contínuo mesmo sendo oferecida a melhor qualidade ao cliente; ter reação rápida à mudança: detectar o problema e solucioná-lo; decidir a mudança por meio de dados, e não por meio de empirismo; ter controle sobre os processos para evitar maus resultados. (FALCONI, 2004).

Estar inserido no Programa de Gestão de Qualidade Total permite o estímulo para a procura da melhoria contínua da qualidade da assistência prestada. Todos os enfermeiros devem compreender o sentido da acreditação, pois uma empresa acreditada demonstra credibilidade para com toda a comunidade, e consegue esse êxito por meio de padrões definidos (LIMA et al., 2000).

$\mathrm{Na}$ abordagem fundamentada nos princípios desse Programa faz-se necessário investir na capacitação do profissional gerando certa satisfação profissional para atender bem aos clientes, ou seja, a exemplo o enfermeiro: se o enfermeiro sente-se capacitado, satisfeito profissionalmente, será prazeroso satisfazer o cliente interno e o cliente externo, não uma espécie de obrigatoriedade (LIMA et al., 2000).

No serviço de enfermagem, a gestão da qualidade da assistência envolve conhecimento da ciência da enfermagem, habilidades, crenças, valores individuais, 
profissionais e institucionais, ou seja, torna-se imperativo que todos se empenhem em fazer uso adequado dos recursos tanto materiais quanto humanos voltados para oferecer o melhor possível aos seus clientes (LIMA et al., 2000).

O enfermeiro que gerencia o serviço da assistência de enfermagem tem autonomia para identificar os problemas, sugerir melhorias e aplicar as correções necessárias a fim de manter, melhorar ou até mesmo modificar a assistência ao cliente (FELDMAN; GATTO; CUNHA, 2005).

Deve se tornar claro para a instituição o papel do enfermeiro, pois se este não for entendido, não haverá a autonomia, e sem ela fica difícil se concretizarem as práticas da qualidade, pois, já que é a enfermagem que lida continuamente com o paciente, é ela quem mais contribuirá para a resolução dos problemas (COCK; HIPKIN, 1997).

Além disso, o enfermeiro tem grande contribuição na elevação do nível de educação e treinamento dos funcionários, e pode promover um relacionamento aberto e a integração de conhecimentos e competências (FELDMAN; GATTO; CUNHA, 2005).

É importante ressaltar que os enfermeiros assistenciais podem sempre oferecer sugestões, porém, é a alta gerência que deve se responsabilizar pela visão estratégica na organização, estabelecendo as diretrizes e os controles estatísticos, unindo teoria à prática, tendo assim uma visão geral para solucionar os problemas organizacionais. (DEI SVALDI; LUNARDI FILHO; GOMES, 2006). Existem hospitais que se inserem nos programas de gestão, mas que não realizam uma mudança de cultura. Isso faz com que os enfermeiros não consigam se envolver de forma adequada com o trabalho (COCK; HIPKIN, 1997).

Há uma grande dificuldade em obter qualidade em saúde, principalmente na enfermagem que é a equipe mais numerosa e que passa maior tempo com o paciente, pois só se consegue obter a qualidade, propriamente dita, quando a empresa torna-se competitiva, e o espírito de competitividade deve ser moldado em cada um da equipe (CORRÊA et al, 2007). 
Quando é citada a questão da competitividade, está relacionado a cada integrante da empresa saber o significado de todos os processos, para entender o que envolve um bom resultado. É sentir orgulho de trabalhar em um lugar onde o maior objetivo de todos é a satisfação de alta qualidade (CORRÊA et al, 2007).

Os enfermeiros devem ter autoridade e capacitação ao preparar sua equipe para atender aos clientes com excelência, além de proporcionar uma harmonia entre os trabalhadores e a organização. Por isso, os processos devem ser bem estruturados, deve haver projetos e planos com visão de futuro (CORRÊA et al., 2007).

Investir em qualidade faz com que os defeitos sejam minimizados, proporciona uma melhora dos produtos, os lucros são maiores e os prejuízos quase zero, proporciona maior bem-estar, os funcionários permanecem por maior tempo na empresa, os clientes encontram-se cada vez mais satisfeitos (LEITÃO; NASCIMENTO; VARGENS, 2006).

Essa investidura dá liberdade para os funcionários falarem o que têm necessidade para a alta gerência, porém, fica entendido que qualquer extremismo é ruim. Deve haver um equilíbrio para que não haja fanatismo e nem desleixo por parte dos integrantes da equipe (COLE, 1998).

Para Donabedian (1990), ao se pensar em avaliação da qualidade, devem ser levados em consideração os sete pilares da qualidade: eficácia, efetividade, eficiência, otimização, aceitabilidade, legitimidade e equidade. Segundo o autor, para se avaliar a assistência à saúde, faz-se necessário ter em mente esses pilares, pois eles são a base para quem quer alcançar a qualidade.

Para se obter um nível de qualidade excelente, tanto a estrutura, quanto o processo e o resultado não devem ser considerados como elementos autônomos, pelo contrário, eles estão intimamente relacionados e mantêm certa linha de causalidade e efeito que é preciso para se avaliar o cuidado (MEZOMO, 1995).

Ainda se falando em estrutura, devem ser avaliadas as características dos recursos que empregam a atenção médica e de enfermagem, a organização admi- 
nistrativa, a descrição das instalações, o perfil dos profissionais, considerando se os mesmos se encontram preparados e experientes (REIS et al., 1990).

Agora, em se tratando de Processo, para Corrêa (2007, p. 3), “[...] são as atividades de cuidados realizadas para um paciente, ligadas a um resultado, para atingirem suas metas".

Para gerenciar, é essencial conhecer os processos, pois tanto os resultados bons quanto os ruins, dependem do processo. A gestão pela qualidade total sugere uma subdivisão deles: detectar problemas buscando os resultados indesejáveis; analisar buscando as causas e atuar melhorando os resultados. Para Deming (DEMING apud NOGUEIRA, 1999) 85 a 95\% dos problemas de uma empresa estão nos processos, e não nas pessoas.

Os funcionários devem entender o processo da qualidade, estando cada dia mais motivados e envolvidos com oportunidades de crescimento, pois a empresa depende dos funcionários e não o contrário (MEZOMO, 1995).

O processo dos serviços de enfermagem está subdividido em subprocessos,

[...] que são denominados cuidar ou assistir, administrar ou gerenciar, pesquisar e ensinar" (FELLI; PEDUZZI, 2005, p. 6). A ação do trabalho do enfermeiro divide-se em dois processos: o processo "cuidar" e o processo "administrar" (FELLI; PEDUZZI, 2005).

Quando falamos em processo administrar, na enfermagem, estamos vivenciando a organização do trabalho e os recursos humanos da área, ou seja, o enfermeiro nesse momento realiza o planejamento, o dimensionamento de pessoas do setor, seleciona e recruta o pessoal,, realiza a educação continuada/permanente, supervisiona, avalia o desempenho de sua equipe, entre outros (FELLI; PEDUZZI, 2005).

O Processo do cuidar está voltado à satisfação das necessidades de saúde da população, o que requer envolvimento do cuidado tanto dos usuários, quanto dos trabalhadores que estão proporcionando esses serviços (CAMPOS, 1994). 
A equipe de enfermagem é como o nome já diz, uma equipe, e está ligada diretamente aos cuidados com o paciente, sendo assim, precisa de alguém (enfermeiro) para estar coordenando as suas ações, para que haja um acordo comum e todos proporcionem uma assistência integral e de excelência ao paciente (MARX, 1990).

Com isso, o processo do cuidar é o processo de trabalho específico, ou seja, na enfermagem é o cuidado assistencial em si. Ele envolve o que está sendo feito pelo e para o paciente de modo a assisti-lo bem. No trabalho de enfermagem, pode estar sendo avaliado o que é feito para que o paciente não adquira úlcera por pressão, ou se a equipe de enfermagem está administrando o medicamento no horário certo, ou ainda, se a equipe de enfermagem está realizando uma punção venosa periférica com a técnica correta, entre outros (SILVA, 1996).

Ainda se tratando disso, o enfermeiro deve ter uma visão global sobre tudo o que está acontecendo em seu ambiente de trabalho, pois é necessário que haja indicadores para detectar os problemas para resolvê-los (SILVA, 1996). Os indicadores possibilitam o monitoramento da qualidade dos serviços a fim de melhorar cuidado oferecido (MOURA et al., 2009).

Para Donabedian (1994) a melhor forma de avaliar os processos é utilizar levantamento de dados, e, normalmente, eles são detectados em forma de indicadores de enfermagem, como detalhados a seguir.

Indicadores são: "Especificação quantitativa e qualitativa para medir o alcance de um objetivo" (FINANCIADORA DE ESTUDOS E PROJETOS, 2002, p. 2).

Para que os indicadores sejam validados, é necessário que se atenha sempre à mesma norma ou forma de medida, permitindo, desse modo, a comparabilidade. Um importante aspecto que deve ser atentado é que, para que os indicadores sejam realmente ferramentas úteis, eles devem ser produzidos com regularidade, permitindo visualizar as tendências do tempo e nos dados, sem esquecer que estes devem ser publicados para que qualquer pessoa tenha acesso de forma facilitada (ROZADO, 2005). 
Segundo Carvalho (2003), os indicadores padronizados para a gestão hospitalar são: indicadores com ênfase nos usuários (satisfação do cliente); indicadores relacionados aos recursos humanos (absenteísmo, índice de frequência de acidentes de trabalho, hora de treinamento por trabalhador); indicadores com foco nos pacientes (tempo de permanência no hospital, índice de infecção hospitalar) e indicadores com foco na administração (taxa de ocupação de leitos, margem líquida).

Os principais indicadores de enfermagem são: estado de conservação e funcionamento de equipamentos; registro de entrada e saída de fluidos corpóreos do paciente; registro de peso diário e/ou eliminações fisiológicas; índice de satisfação do cliente; administração de medicamentos (erros, pontualidade, checagem, identificação); qualidade da assistência baseada em diagnósticos de enfermagem; participação efetiva da equipe nos treinamentos elaborados .(VARGAS et al., 2007).

Além desses, existem o coeficiente de queda de pacientes, coeficiente de infecção hospitalar, índice de cuidados com acesso venoso central e periférico, incidência de úlcera por pressão, procedimentos ou terapias canceladas devido à preparação inadequada do paciente, documentação da enfermagem incompleta, proporção de extubação acidental, proporção de Perda de Sonda Nasoenteral para aporte nutricional, entre outros (VARGAS et al., 2007).

Porém, já que controlar com êxito um processo não é fácil, foi necessário adotar um método, com ferramentas técnicas específicas para conseguir alcançar os objetivos almejados, esse método é o ciclo PDCA (Plan, Do, Check e Act) de controle de processos (NOGUEIRA, 1999).

P (Plan) é quando as metas são estabelecidas, é o momento do planejamento. Essa fase é o período em que é encontrado o problema, e são definidos os métodos para alcançar a meta desejada. D (Do) é a fase de execução do planejamento, é o momento em que se educa e se coleta dados. C (Check) é a verificação dos resultados, a análise dos dados coletados. A (Act) é a fase em que se atua, se o resultado foi bom, o método é padronizado, se foi ruim, é preciso atuar corretivamente (NOGUEIRA, 1999). 
O ciclo PDCA é utilizado para manutenção do nível de controle da qualidade, é também utilizado nas melhorias do nível de controle. O caminho do sucesso para se obter melhorias contínuas no processo é o de utilizar o PDCA tanto para manutenção quanto para melhorias (FALCONI, 2004).

Por fim, será abordado o Resultado, que é entendido como o que é alcançado pelo e para o usuário, seja ele um indivíduo, um grupo ou uma população. Donabedian (1990, p. 72) conceitua: "Resultado é o produto final da assistência prestada, considerando saúde, satisfação de padrões e de expectativas".

Logo, o principal objetivo do resultado é o nível de satisfação do cliente, se o resultado encontrado for bom, a estrutura e o processo devem ser padronizados, porém, se for ruim, pode ser realizado um plano estratégico para melhorar a situação, como por exemplo, o plano de ação corretiva (FALCONI, 2004).

Plano de ação corretiva é um conjunto de ações realizadas para ser melhorado o resultado. Cada pessoa, em cada nível hierárquico, deverá ter seu próprio Plano de Ação. Para montar esse plano, devem ser respondidas seis perguntas: $\mathrm{O}$ que está acontecendo? Quem é o responsável? Quando melhoraremos? Onde está acontecendo o problema? Por quê? E como? Depois de respondidas essas perguntas, deve ser elaborado um plano de ações permanentes com padronização, para que não se repita o resultado ruim (FALCONI, 2004).

\section{Considerações finais}

Um entre tantos outros papéis dos enfermeiros é o de cuidar, que dá conotação de cuidado e zelo. É acompanhar, assistir, auxiliar, proteger, é dar atenção, ou seja, satisfazer as necessidades do cliente em sua totalidade (PAIVA, 2006).

O Processo do trabalho dos serviços de enfermagem demanda dos enfermeiros um conhecimento que vai além da assistência. Os enfermeiros devem saber planejar, ter visão crítica para saberem identificar onde estão os problemas, estabelecer prioridades para promover e recuperar a saúde do paciente com qualidade (PAIVA, 2006). 
Os serviços de enfermagem devem ser continuados, ou seja, a melhoria deve ser contínua. Toda a equipe de enfermagem que trabalha em um determinado lugar do hospital conhece o sistema de funcionamento da unidade, os erros e as qualidades. Sendo assim, todos podem estar oferecendo melhoria para os serviços, pois os serviços sempre necessitarão de reavaliações (CHIAVENATO, 2006).

A equipe de enfermagem presta assistência ao usuário durante as 24 horas do dia, logo, dois fatores devem ser considerados. Primeiro, é fundamental que a enfermagem se envolva com o programa de gestão pela qualidade total já que, dentro do hospital, é a maior equipe, maior contingente de pessoas. O segundo é que a enfermagem é quem mantém o maior contato direto e contínuo com o cliente externo, necessitando, desse modo, ultrapassar as expectativas do cliente durante sua permanência no hospital (ANTUNES, 1997).

Sendo assim, torna-se visível a importância da equipe de enfermagem. Por esse motivo, todos os membros da equipe devem ser treinados, melhorando o desempenho técnico, o atendimento ao paciente e aos familiares, além de educar-lhes quanto à importância da filosofia da qualidade, introduzindo a mudança da cultura e garantindo, as condições de realização profissional e pessoal dos trabalhadores (ANTUNES, 1997).

Os enfermeiros, quando pensarem em qualidade, devem estar atentos ao conhecimento técnico-científico, aos bons materiais, equipamentos e profissionais capacitados; às rotinas de serviços, definindo-as bem; devem avaliar os serviços e, fazerem parte da equipe de profissionais que tenham atitudes e comportamentos que visem à satisfação das necessidades dos clientes (SILVA; PINHEIRO, 2001).

Pensar que a equipe de enfermagem, particularmente nos hospitais, é a que fica a maior parte do tempo com o paciente, faz com que seja questão de sobrevivência adotar o Programa de gestão pela qualidade total. $\mathrm{O}$ cliente que tem a oportunidade de utilizar os serviços de saúde com qualidade zela pela empresa e não a troca por nenhuma outra. 


\section{Management of quality in a hospital nursing service}

\section{Abstract}

This paper aims speaking about some of the factors of quality, in terms of structure, process and outcome of nursing services at the hospital, which influence in quality of care provided to the citizens. It is a bibliographic and descriptive study that used scientific articles published since 1990 up to 2009. The quest for quality has always been present, the reason for industrial companies to opt for a quality service was the competition between them. The Total Quality Management program was created to help companies to manage quality. Hospitals are evaluated by the Joint Commission on Accreditation of Hospitals and the National Accreditation Organization. Both make evaluations according to the proposed by Donabedian: structure, process and outcome. The accreditation includes nursing quality assistence evaluation.

Keywords: Management. Quality. Nurse. Quality assessment. User satisfaction.

\section{Referências}

ANTUNES, A.V. O gerenciamento da qualidade na enfermagem. 1997. Tese (Doutorado em Enfermagem Fundamental)-Escola de Enfermagem de Ribeirão Preto, Universidade de São Paulo, Ribeirão Preto, 1997.

ANTUNES, A. V.; TREVIZAN, M. A. Gerenciamento da qualidade: utilização no serviço de enfermagem. Revista Latino Americana de Enfermagem, Ribeirão Preto, v. 8, n. 1, p. 35-44, jan. 2000.

BARROS, C. S. Avaliação da qualidade dos hospitais públicos baianos segundo o modelo de gestão. 135f. Dissertação (Mestrado)-Programa de Pós-Graduação da Escola de Enfermagem da Universidade Federal da Bahia (UFBA), Salvador, BA, 2005.

BERWICK, D. M.; GODFREY, A. B.; ROESNER, J. Melhorando a qualidade dos serviços médicos, hospitalares e da saúde. São Paulo: Makron Books, 1994. 
CAMPOS, G. W. S. Considerações sobre o processo de administração e gerência de serviços de saúde. In: CAMPOS, W. G. S.; MERHY, E. E.; NUNES, E. D. (Org.) Planejamento sem normas. 2. ed. São Paulo: Hucitec, 1994.

CARVALHO, M. A. Indicadores de avaliação de desempenho em estabelecimentos assistenciais de saúde. In: ANAIS do Fórum permanente e interdisciplinar de saúde. Campinas: Unicamp, 2003.

CHIAVENATO, I. Administração: teoria, processo e prática. 3. ed. São Paulo: Makron Books, 2006.

COCK, C.; HIPKIN, I. TQM and BPR: beyond the beyond myth. Journal of Management Studies, Oxford, v. 34, n. 5, p. 659-675, 1997. doi:10.1111/14676486.00067 .

COLE, R. E. Learning from the Quality Movement: what did and didn't happen and why? California Management Review, Los Angeles, v. 41, n. 1, p. 43-73, 1998.

CORREAA, I. R. S. et al. Indicadores de qualidade do serviço de enfermagem: administração de enfermagem. São Paulo: Universidade de Uberaba, 2007.

DONABEDIAN, A. The seven pilares of quality. Archives of pathology \& Laboratory Medicine, Northefiel, v. 114, n.11, p. 1115, 1990.

DONABEDIAN, A. La calidad de la atención médica: definición y métodos de evaluación. México: Copilco, 1991.

DONABEDIAN, A. A gestão da qualidade total na perspectiva dos serviços de saúde. Tradução de Roberto Passos Nogueira. Rio de Janeiro: Qualitymark, 1994.

FALCONI, V. C. Gerenciamento pelas diretrizes. Nova Lima, MG: INDG Tecnologia e Serviços, 2004.

FELDMAN, L. B.; GATTO, M. A. F.; CUNHA, I. C. K. O. História da evolução da qualidade hospitalar: dos padrões à acreditação. Acta Paulista de Enfermagem, São Paulo, v. 18, n. 2, p. 213-219, abr./jun. 2005. doi: 10.1590/S010321002005000200015.

FELLI, V.; PEDUZZI, M. O Trabalho gerencial em enfermagem. In: KURCGANT, Paulina (Org.). Gerenciamento em enfermagem. Rio de Janeiro: Guanabara Koogan, 2005. v. 1. p. 1-13. 
FINANCIADORA DE ESTUDOS E PROJETOS (FINEP). Termos e Conceitos. Rio de Janeiro, 2002. Disponível em: <http://www.finep.gov.br/empresa/conceitos_ ct.asp?>. Acesso em: 29 dez. 2008.

KURCGANT, P. et al. Gerenciamento em enfermagem. 2. ed. Rio de Janeiro: Guanabara Koogan, 2005.

LIMA, S. B. S.; ERDMANN, A. L. A enfermagem no processo de acreditação hospitalar em um serviço de urgência e emergência. Acta Paulista de Enfermagem, São Paulo, v. 19, n. 3, p. 271-278, set. 2006. doi:10.1590/S0103-21002006000300003.

LIMA, S. B. S. et al. O processo de acreditação hospitalar e a enfermagem. Acta Paulista de Enfermagem, São Paulo, v. 13, p. 190-196, 2000.

MARX, K. Obras escolhidas. 2. ed. Lisboa: Avante, 1990.

MELLO, J. B.; CAMARGO, M. O. Qualidade na saúde práticas e conceitos: normas ISO nas áreas médico-hospitalar e laboratorial. São Paulo: Best Seller, 1998.

MEZOMO, J. C. Gestão da qualidade na saúde: princípios básicos. 3. ed. São Paulo: Câmara Brasileira do Livro, 1995.

MOURA G. M. S. S. et al. Construção e implantação de dois indicadores de qualidade assistencial de enfermagem. Revista Gaúcha de Enfermagem, Porto Alegre, v. 30, n. 1, p.136-140, mar, 2009.

NASCIMENTO, I. J.; LEITÃO, R. E. R.; VARGENS, O. M. C. A qualidade nos serviços de saúde pública segundo enfermeiros que gerenciam unidades básicas de saúde. Revista Enfermagem UERJ, Rio de Janeiro, v. 14, n. 3, p. 350-356, jul./ set. 2006 .

NOGUEIRA, L. C. Gerenciando pela qualidade total na saúde. 2. ed. Belo Horizonte: Desenvolvimento Gerencial, 1999.

NONINO, E. A. P. M. Avaliação da qualidade dos procedimentos de enfermagem: banho e curativo: segundo grau de dependência assistencial dos pacientes internados em um hospital universitário. 2006. Tese (Doutorado)-Escola de Enfermagem de Ribeirão Preto, Ribeirão Preto, 2006.

OLIVEIRA, O. J. et al. Gestão da qualidade: tópicos avançados. São Paulo: Pioneira Thompson Learning, 2004. 
PAIVA, S. M. M. Avaliação da qualidade de vida de paciente oncológicos em tratamento quimioterápico adjuvante. Dissertação (Mestrado)-Escola de Enfermagem de Ribeirão Preto, Universidade de São Paulo, Ribeirão Preto, 2006.

REIS, E. J. F. B. et al. Avaliação da qualidade dos serviços de saúde. Caderno de Saúde Pública, Rio de Janeiro, v.6, n. 1, p. 50-61, jan./mar. 1990. doi:10.1590/ S0102-311X1990000100006.

ROZADO, H. B. F. Uso de indicadores na gestão de recursos de informação. Revista Digital de Biblioteconomia e Ciência da Informação, Campinas, v. 3, n. 1, p. 60-76, jul./dez. 2005.

SILVA, V. F. O desgaste do trabalhador de enfermagem: a relação trabalho de enfermagem e saúde do trabalhador. Tese (Doutorado)-Escola de Enfermagem USP, São Paulo, 1996.

SILVA, M. J. P.; PINHEIRO, E. M. Qualidade na assistência de enfermagem: visão de alunas de especialização. Acta Paulista de Enfermagem, São Paulo, v. 14, n. 1, p. 82-88, jan./abr. 2001.

SVALDI, J. S. D.; LUNARDI FILHO, W. D.; GOMES, G. C. Apropriação e uso de conhecimento de gestão para mudança de cultura na enfermagem como disciplina. Texto \& Contexto - Enfermagem, Florianópolis, v. 15, n. 3, p. 500-507, 2006. doi: 10.1590/S0104-07072006000300016.

VARGAS et al. Onde e quando encontramos qualidade nos serviços de enfermagem hospitalar. Revista Brasileira de Enfermagem, Brasília, v. 60, n. 3, p. 339-343, maio/jun. 2007. doi:10.1590/S0034-71672007000300018. 
Para publicar na revista Universitas: Ciências da Saúde, acesse o endereço eletrônico www.publicacoesacademicas.uniceub.br. Observe as normas de publicação, para facilitar e agilizar o trabalho de edição. 